\title{
MICROFLOTAÇÃO DE APATITA UTILIZANDO ÓLEO DA CASTANHA DE MACAÚBA (ACROCOMIA ACULEATA) COMO COLETOR
}

\author{
André Carlos Silva ${ }^{\prime}$ \\ Elenice Maria Schons Silva ' \\ Tércio William Pereira Rocha'
}

\section{Resumo}

A macaúba (nome científico Acrocomia Aculeata) é uma palmeira típica do cerrado brasileiro de grande interesse socioeconômico por sua alta capacidade para a produção de óleo vegetal com elevados teores de ácidos graxos. O presente trabalho avaliou a viabilidade técnica da utilização do óleo da castanha de macaúba saponificado (OCMS) como coletor na microflotação de apatita em tubo de Hallimond, sendo o óleo caracterizado quimicamente e saponificado antes de sua aplicação nos ensaios. Foram realizados ensaios variando-se o pH da solução $(8,9$ e 10$)$ e a concentração do coletor $(2,5,5,0,7,5$ e $10 \mathrm{mg} / \mathrm{L})$. De modo a comparar os resultados com o OCMS utilizou-se o coletor industrial Flotigam 5806 $\mathrm{da}$ Clariant nas mesmas condições testadas. Os resultados obtidos mostram que o OCMS alcançou maiores recuperações de apatita nos pH's 8 e 9, sobretudo nas concentrações 7,5 e 10,0 mg/L. Quando comparado ao Flotigam 5806, o OCMS apresentou recuperações menores em todos os pH's e concentrações testados.

Palavras-chave: Flotação; Óleo da castanha de macaúba; Apatita.

\section{APATITE'S MICROFLOTATION USING MACAÚBA'S (ACROCOMIA ACULEATA) NUT OIL AS COLLECTOR}

\begin{abstract}
Macaúba (Acrocomia Aculeata) is a palm tree typical of the Brazilian savanna with great socioeconomic interest due to its high capacity of vegetable oil production with high levels of fatty acids. This paper evaluated the technical viability of saponified macaúba's nut oil (SMNO) as a collector in apatite's micro flotation in Hallimond tube, being the oil chemically characterized and saponified before its utilization. Tests were performed varying the solution $\mathrm{pH}(8,9$ and $\mathrm{I} 0)$ and collector concentration $(2.5,5.0,7.5$ e $10 \mathrm{mg} / \mathrm{L})$. To compare the obtained results with SMNO the industrial collector Clariant's Flotigan 5806 was used in the same tests conditions. Results shows that SMNO reached the highest recoveries rates of apatite in $\mathrm{pH} 8$ and 9, particularly at the concentrations 7.5 and $10 \mathrm{mg} / \mathrm{L}$. When compared to Flotigam 5806, the MNO showed lower recoveries at all pHs and concentrations tested.
\end{abstract}

Keywords: Froth flotation; Macaúba's nut oil; Apatite.

\section{INTRODUÇÃO}

Segundo Fonseca [I] dados do U.S. Geological Survey (USGS) apontam que a produção mundial de fosfato no ano de 2013 foi de 223.585 t (aumento de $3,1 \%$ em relação à 2012), superando a produção de todos os anos anteriores. A China liderou a produção mundial, com 43,4\% do total, seguida, em menor escala, por Estados Unidos, Marrocos, Rússia e Brasil. Sendo que apenas três países, China, Estados Unidos e Marrocos (incluindo Saara Ocidental), foram responsáveis por $70 \%$ da produção mundial. Já a produção brasileira em 2013 foi de $6.715 \mathrm{t}$.

Souza e Fonseca [2] afirmam que no Brasil, cerca de $80 \%$ das jazidas fosfáticas naturais (fosfatos), são de origem ígnea com presença acentuada de rocha carbonatítica e minerais micáceos, com baixo teor, variando de $4 \%$ a I5\% de pentóxido de fósforo $\left(\mathrm{P}_{2} \mathrm{O}_{5}\right)$, enquanto que em termos mundiais esse percentual está em torno de 17\%. A maior parte dos minerais de fósforo destas rochas pertence

'Universidade Federal de Goiás - UFG, Catalão, GO, Brasil. E-mail: ancarsil@ufg.br 
ao grupo da apatita, representado pela fórmula química $\mathrm{Ca}_{5}(\mathrm{~F}, \mathrm{Cl}, \mathrm{OH})\left(\mathrm{PO}_{4}\right)_{3}$.

Para Monte e Peres [3] os principais depósitos de fosfatos brasileiros (de origem ígnea) apresentam como diferença básica em relação aos da Flórida e de Marrocos (de origem sedimentar), por exemplo, a presença de ganga carbonática associada à apatita. Esta peculiaridade exigiu a definição de esquemas de reagentes específicos, a qual considerava o uso de tall oil como coletor de apatita e amido de milho convencional como depressor de minerais de ganga. Desde então, diversos trabalhos têm sido realizados a fim de se encontrar novos reagentes de flotação, com destaque para os coletores e depressores.

A finalidade básica do coletor é formar seletivamente uma camada hidrofóbica sobre a superfície mineral e, dessa forma, possibilitar condições para a fixação das partículas hidrofobizadas às bolhas de ar e consequente recuperação destas partículas na espuma. De acordo com Baltar [4] os coletores podem ser aniônicos (sulfidrílicos ou oxidrílicos) ou catiônicos (aminas e seus sais). Dentre os oxidrílicos, pode-se destacar a família dos carboxílicos, que contém os ácidos graxos, que apresentam grande destaque na literatura como coletores no processo de flotação. Segundo Bulatovic [5] estes podem ser encontrados em óleos de origem vegetal e animal.

Para Abdel-Khalek et al. [6], os ácidos graxos são os coletores mais usados em flotação direta de minérios de fosfato. Ácidos graxos de cadeias longas e os seus sais são os mais extensivamente utilizados, sobretudo o ácido oleico saponificado (ou oleato de sódio). Guimarães et al. [7] lembram que a crença no potencial coletor superior do ácido oleico remonta ao início da flotação de fosfatos, em que se realizavam estudos para encontrar coletores alternativos ao tall oil.

Diversos estudos têm sido realizados com o intuito de se identificar óleos vegetais que ofereçam novas opções de coletores aos já disponíveis no mercado. Dentre estes pode-se citar o trabalho de Costa [8], que estudou óleos vegetais da Amazônia na flotação de apatita, calcita e quartzo. Foram utilizadas as polpas de açaí, buriti e inajá, as sementes de andiroba e maracujá e a castanha do Pará (a composição de ácidos graxos dos óleos é apresentada na Tabela I). Os resultados obtidos pelo autor demonstraram que o buriti, inajá, andiroba e açaí apresentaram curvas semelhantes de recuperação da apatita em função da concentração de coletor e atingiram a máxima recuperação de apatita (próximo de 100\%) na concentração de 2,5 mg/L. Já o maracujá e a castanha do Pará, para atingirem essa mesma recuperação, requereram uma concentração de 5,0 mg/L. Nesta concentração $(5,0 \mathrm{mg} / \mathrm{L})$, o maracujá foi o coletor que teve maior recuperação de calcita (em torno de 50\%), talvez pelo fato deste óleo possuir alto teor de ácido linoleico $(48,82 \%)$. Na concentração de $2,5 \mathrm{mg} / \mathrm{L}$ o açaí foi o coletor que apresentou maior seletividade entre apatita e calcita. Nenhum dos coletores apresentou recuperação significativa de quartzo.

Alves et al. [9] realizaram um estudo utilizando o óleo de babaçu e da semente de maracujá como coletores na flotação da apatita, calcita e quartzo. Para os dois óleos não houve recuperação significativa do mineral quartzo. O óleo de maracujá apresentou maior seletividade que o babaçu entre a apatita e a calcita. Enquanto o coletor de maracujá atingiu a flotabilidade máxima da apatita na concentração de $5 \mathrm{mg} / \mathrm{L}$, o coletor de babaçu atingiu a mesma flotabilidade com o dobro dessa concentração ( $10 \mathrm{mg} / \mathrm{L})$. Isto pode ser atribuído ao fato de o óleo de maracujá possuir uma predominância de ácidos graxos insaturados em sua molécula e o óleo de babaçu possuir teores significativos de ácidos graxos saturados (45\% de ácido láurico e 16\% de ácido mirístico).

O cerrado brasileiro apresenta uma enorme variedade de espécies vegetais oleaginosas que ainda não foram estudadas. A palmeira da macaúba, típica deste bioma, pode ser encontrada em extensas áreas do território brasileiro. Esta espécie oferece utilidades em diversas áreas, tais como medicinal, alimentícia, cosmética etc. e tem despertado grande interesse socioeconômico por ser considerada uma das espécies com maior potencial de exploração econômica imediata em solo brasileiro. Tal fato deve-se à sua elevada capacidade de produção de óleo, bem como ao aproveitamento total dos coprodutos gerados. Além disso, seu sistema de cultivo é tido como sustentável do ponto de vista ambiental, social e econômico. O presente trabalho visou avaliar a viabilidade técnica da utilização do óleo da

Tabela I. Composição de ácidos graxos óleos vegetais da Amazônia

\begin{tabular}{|c|c|c|c|c|c|c|}
\hline \multirow{2}{*}{$\begin{array}{c}\text { Ácidos Graxos } \\
\text { (\%) }\end{array}$} & \multicolumn{6}{|c|}{ Óleos Vegetais } \\
\hline & Açaí & Andiroba & Buriti & Castanha & Inajá & Maracujá \\
\hline Esteárico & 2,07 & 10,27 & 2,17 & 13,17 & 3,19 & 5,04 \\
\hline Láurico & - & - & - & - & 4,46 & - \\
\hline Linoleico & 7,58 & 5,4 & 7,03 & 15,2 & 9,4 & 48,82 \\
\hline Linolênico & 0,22 & - & 1,08 & - & $\mathrm{I}, 44$ & 0,19 \\
\hline Mirístico & 0,26 & - & - & - & 8,26 & 0,19 \\
\hline Oleico & 61,55 & 51,47 & 70,55 & 47,02 & 49,9 & 28,94 \\
\hline Palmítico & 21,75 & 31,28 & 17,44 & 18,13 & 21,27 & 12,56 \\
\hline
\end{tabular}

Fonte: Adaptado de Costa [8] 
castanha da macaúba saponificado (OCMS) como coletor na microflotação de apatita em tubo de Hallimond. Uma vez que este novo reagente de flotação pode ser obtido a partir de fontes sustentáveis, o seu uso pode contribuir com a preservação do meio ambiente, além de gerar novas fontes de renda e emprego para produtores deste fruto.

\section{MATERIAIS E MÉTODOS}

As amostras de apatita utilizadas nos ensaios de microflotação em tubo de Hallimond foram adquiridas em granulometria acima de $1,0 \mathrm{~cm}$ e, para a adequação granulométrica das mesmas, estas foram submetidas à moagem em moinho de bolas e, posteriormente, realizou-se o peneiramento a úmido. Para a remoção de eventuais contaminações com material magnético na etapa de cominuição as amostras foram submetidas a uma separação magnética utilizando um ímã de terras raras com campo de $2.000 \mathrm{G}$.

De modo a estimar a pureza da amostra de apatita foi determinada a sua massa específica utilizando-se um picnômetro de $100 \mathrm{~mL}$ de volume interno. A análise química das amostras foi realizada em um espectrômetro de fluorescência de raios-X da Panalytical, modelo AXIOX MAX Série DY $n^{\circ} 500$ I, na empresa Anglo American Fosfatos do Brasil, localizada nos municípios de Catalão e Ouvidor/GO, sendo analisados apenas os seguintes óxidos: $\mathrm{P}_{2} \mathrm{O}_{5}, \mathrm{Fe}_{2} \mathrm{O}_{3}$, $\mathrm{SiO}_{2}, \mathrm{BaO}, \mathrm{Al}_{2} \mathrm{O}_{3}$ e $\mathrm{CaO}$.

O óleo de castanha de macaúba (OCM) utilizado nos ensaios de microflotação foi produzido pela Associação Riacho Danta Macaúba (situada na zona rural de Montes Claros/MG) por prensagem mecânica a frio e caracterizado utilizando-se um cromatógrafo a gás, modelo HP5890, da UFMG, equipado com detector por ionização de chamas. Utilizou-se uma coluna SP2380 (Supelco) de $30 \mathrm{~m} \times$ $0,25 \mathrm{~mm} \times 0,20 \mu \mathrm{m}$ com gradiente de temperatura de $120^{\circ} \mathrm{C}$ em I min e de $7^{\circ} \mathrm{C} / \mathrm{min}$ até $220^{\circ} \mathrm{C}$; injetor (split de $\mathrm{I} / 50$ ) a $250^{\circ} \mathrm{C}$ e detector a $250^{\circ} \mathrm{C}$. Como gás de arraste foi utilizado hidrogênio com uma vazão de $2 \mathrm{~mL} / \mathrm{min}$ e volume de injeção de $2 \mu \mathrm{L}$. A identificação dos picos foi feita por comparação com padrões de ácidos graxos metilados (SUPELCO37).

A caracterização química do OCM foi desenvolvida a partir dos procedimentos do Instituto Adolfo Lutz [10], que se fundamentam nos métodos da American Oil Chemists' Society (AOCS). Os seguintes índices foram determinados: índice de saponificação (IS), matéria insaponificável (MI), índice de acidez (IA) e índice de iodo (II). Todos os ensaios foram realizados em triplicata.

Antes de ser submetido aos ensaios de microflotação, fez-se necessário que o OCM passasse pelo procedimento de hidrólise alcalina (saponificação), de modo a torná-lo solúvel em água, e, com isso, contribuir para seu melhor desempenho como coletor. A saponificação foi realizada com a utilização de hidróxido de sódio $(\mathrm{NaOH})$ a $10 \%$, seguindo a metodologia proposta pela Clariant para saponificação a frio. Nesta metodologia pesa-se 5,0 g do coletor em um béquer de $150 \mathrm{~mL}$, previamente tarado contendo um bastão de agitação magnética. Adiciona-se 20,0 $\mathrm{g}$ de água destilada, dispondo o conjunto em um agitador magnético. Com a agitação ligada, adiciona-se $7,5 \mathrm{~mL}$ de hidróxido de sódio a 10\% para saponificar a solução. Ao final retorna-se o conjunto para a balança e adiciona-se água destilada até atingir-se um peso total de 100,0 g. Coloca-se novamente o conjunto sob agitação para realizar a homogeneização da solução saponificada. Não houve adição de espumantes para estabilização da espuma, uma vez que o coletor, na faixa de $\mathrm{pH}$ analisada, atua também como espumante.

Os ensaios de microflotação foram desenvolvidos em um tubo de Hallimond modificado fabricado pela Hialoquímica, variando-se a concentração do coletor e $\circ \mathrm{pH}$ da solução. Todos ensaios foram realizados em triplicata e o procedimento operacional, baseado em Costa [8], consistia em pesar a amostra de apatita $(\mathrm{I}, 0 \mathrm{~g})$ na faixa granulométrica de $-150+106 \mu \mathrm{m}(-100+150 \#)$ e introduzi-la no tubo de Hallimond. Na sequência era adicionada a solução coletora com dosagem e pH previamente ajustados. A solução era então condicionada por 7 minutos. Após o condicionamento eram adicionados $320 \mathrm{~mL}$ de água destilada pela parte superior do tubo de Hallimond e iniciada a flotação com a introdução do ar com vazão e pressão conhecidas durante I minuto. O material flotado e afundado eram então coletados e encaminhados para secagem em estufa a $70^{\circ} \mathrm{C}$ por 9 horas, repouso à temperatura ambiente por 3 horas e posterior pesagem. Os parâmetros operacionais adotados nos ensaios de microflotação em Tubo tubo de Hallimond podem ser vistos na Tabela 2.

Para fins de comparação com os resultados obtidos com OCMS foram comparados com os resultados obtidos com Flotigam 5806 da Clariant, que é um coletor desenvolvido para a flotação de rocha fosfática brasileira, composto por uma formulação de ácidos graxos. O Flotigam 5806 foi submetido ao mesmo processo de saponificação e ensaios de microflotação em tubo de Hallimond adotados para o OCMS.

Tabela 2. Variáveis operacionais adotadas nos ensaios de microflotação em tubo de Hallimond

\begin{tabular}{lc}
\hline \multicolumn{1}{c}{ Condições } & Valores \\
\hline Vazão de $\operatorname{ar}\left(\mathrm{cm}^{3} / \mathrm{min}\right)$ & 40 \\
Pressão de ar (psi) & 10 \\
Tempo de condicionamento $(\mathrm{min})$ & 7 \\
Tempo de flotação $(\mathrm{min})$ & $\mathrm{I}$ \\
Massa do mineral $(\mathrm{g})$ & $\mathrm{I}$ \\
Faixa granulométrica $(\mu \mathrm{m})$ & $-150+106$ \\
Solução coletora $(\mathrm{mL})$ & 320 \\
Concentração do coletor $(\mathrm{mg} / \mathrm{L})$ & 2,$5 ; 5 ; 7,5 ; 10$ \\
$\mathrm{pH}$ & $8 ; 9 ; 10$ \\
\hline
\end{tabular}




\section{RESULTADOS E DISCUSSÃO}

A massa específica das amostras, obtida pelo método do picnômetro foi de $3,198 \mathrm{~g} / \mathrm{cm}^{3}$, cujo resultado confirma o exposto na literatura, em que consta que a massa específica da apatita pura varia entre 3,1 e $3,2 \mathrm{~g} / \mathrm{cm}^{3}$. A Tabela 3 detalha os principais óxidos encontrados e seus respectivos percentuais. Nota-se que a amostra utilizada neste estudo apresenta elevadas concentrações de $\mathrm{P}_{2} \mathrm{O}_{5}$ e $\mathrm{CaO}(92,54 \%)$, constituindo uma amostra com alto grau de pureza.

A Tabela 4 apresenta a composição de ácidos graxos do óleo da castanha de macaúba (OCM) utilizado nos

Tabela 3. Análise química da amostra de apatita por fluorescência de raios-X

\begin{tabular}{ccccccc}
\hline Óxido & $\mathbf{P}_{2} \mathbf{O}_{5}$ & $\mathbf{F e}_{2} \mathbf{O}_{3}$ & $\mathbf{S i O}_{2}$ & $\mathbf{B a O}$ & $\mathbf{A l}_{2} \mathbf{O}_{3}$ & $\mathbf{C a O}_{2}$ \\
\hline$\%$ & 40,50 & 0,07 & 0,94 & 0,06 & 0,38 & 52,04 \\
\hline
\end{tabular}

Tabela 4. Composição de ácidos graxos da castanha de macaúba

\begin{tabular}{|c|c|c|c|c|c|}
\hline Ácido graxo & OCM & Zuppa [I I] & $\begin{array}{c}\text { Hiane et al. } \\
\text { [12] }\end{array}$ & $\begin{array}{c}\text { Amaral et al. } \\
{[13]}\end{array}$ & Fórmulas químicas estruturais \\
\hline Caprílico (C8) & 3,83 & 7,40 & 5,96 & 5,22 & \\
\hline Cáprico (CI0) & 3,29 & 4,40 & $\mathrm{I}, 79$ & 4,56 & \\
\hline Láurico (CI2) & 34,30 & 39,70 & 12,95 & 44,14 & \\
\hline Mirístico (Cl4) & 10,60 & 9,30 & 9,49 & 8,45 & \\
\hline Palmítico $(\mathrm{Cl} 6)$ & 9,09 & 7,60 & 12,62 & 6,57 & \\
\hline Esteárico $(\mathrm{Cl})$ & 4,19 & 2,40 & 6,58 & 2,11 & \\
\hline Araquídico (C20) & - & - & 0,30 & - & \\
\hline Palmitoleico - $\omega 7$ (CI6:I) & - & - & 2,29 & - & \\
\hline Oleico - $\omega 9(\mathrm{CI} 8: \mathrm{I})$ & 31,60 & 25,80 & 40,17 & 25,76 & \\
\hline Linoleico - $\omega 6,9$ (C I8:2) & 3,07 & 2,70 & 5,91 & 3,19 & \\
\hline cis-6-9-octadecadienóico (C 8:2) & - & 0,70 & - & - & \\
\hline Linolênico - $\omega 3,6,9$ (CI8:3) & 0,15 & - & 1,92 & - & $\mathrm{ClI}_{2}-\mathrm{Cll}_{2}-\mathrm{Cll}_{2}$ \\
\hline
\end{tabular}


ensaios de microflotação, bem como análises de três autores diferentes, para comparação. As amostras de macaúba utilizadas por Zuppa [I I] eram oriundas da Universidade Federal de Goiás, Campus Goiânia, sendo que as amostras de Hiane et al. [12] eram oriundas da Universidade Federal de Mato Grosso do Sul e as de Amaral et al. [13] oriundas da Fazenda Experimental Edgardia da UNESP, Campus de Botucatu. Os três autores utilizaram Cromatografia Gasosa de Alta Resolução (HRGC) para a determinação da composição de ácidos graxos da castanha de macaúba. As diferenças nas composições dos ácidos graxos se devem às diferentes localizações do cultivo de cada amostra.

O índice de saponificação, definido como sendo a quantidade de hidróxido de potássio $(\mathrm{KOH})$ necessária para a saponificação dos ácidos graxos em I,0 g de amostra, encontrado para o OCM foi de 202,40 \pm I,69 $\mathrm{mg} \mathrm{KOH} / \mathrm{g}$ de óleo. Este resultado condiz com o obtido por Melo [14], que foi de 192,7 mg KOH /g de óleo.
A matéria insaponificável para o OCM foi de I,6I \pm $0,16 \%$, que mostra que o OCM não contém apenas ácidos graxos, mas também outras substâncias que não puderam ser saponificadas pelo método utilizado.

O valor do índice de acidez obtido para o OCM foi de 16,72 $\pm 0,28 \mathrm{mg}$ de $\mathrm{KOH} / \mathrm{g}$ de óleo, o que sugere que este óleo possui quantidade de ácidos graxos livres em sua composição superior aos encontrados dentre os óleos vegetais de frutos amazônicos, conforme o estudo de Costa [8].

Em relação ao índice de iodo, quando comparado aos resultados dos óleos de frutos amazônicos do estudo de Costa [8], o OCM apresentou valor relativamente baixo $(39,44 \pm 0,42 \mathrm{~g}$ de $\mathrm{l} / \mathrm{g}$ de óleo). A explicação para isso pode estar na composição do OCM que apresenta altas concentrações de ácidos graxos saturados.

As Figuras I a 3 apresentam a recuperação de apatita para os dois coletores (OCMS e Flotigam 5806) em três faixas de $\mathrm{pH}$ e quatro faixas de dosagem. Os resultados obtidos

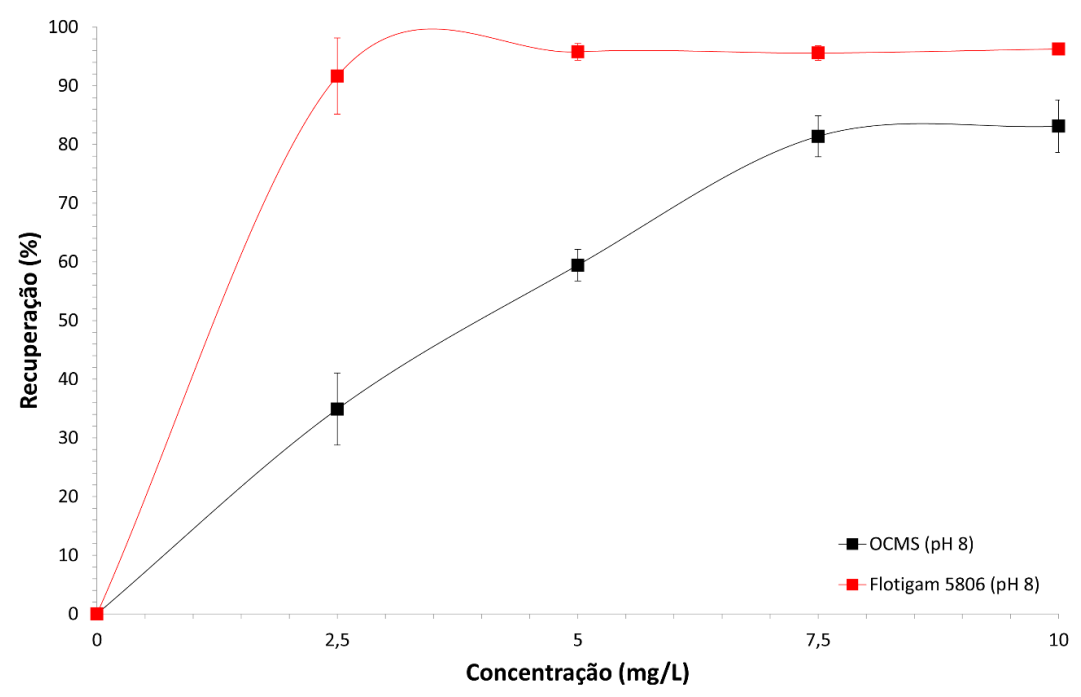

Figura I. Recuperação de apatita em função da dosagem dos coletores OCMS e Flotigam 5806 em pH 8.

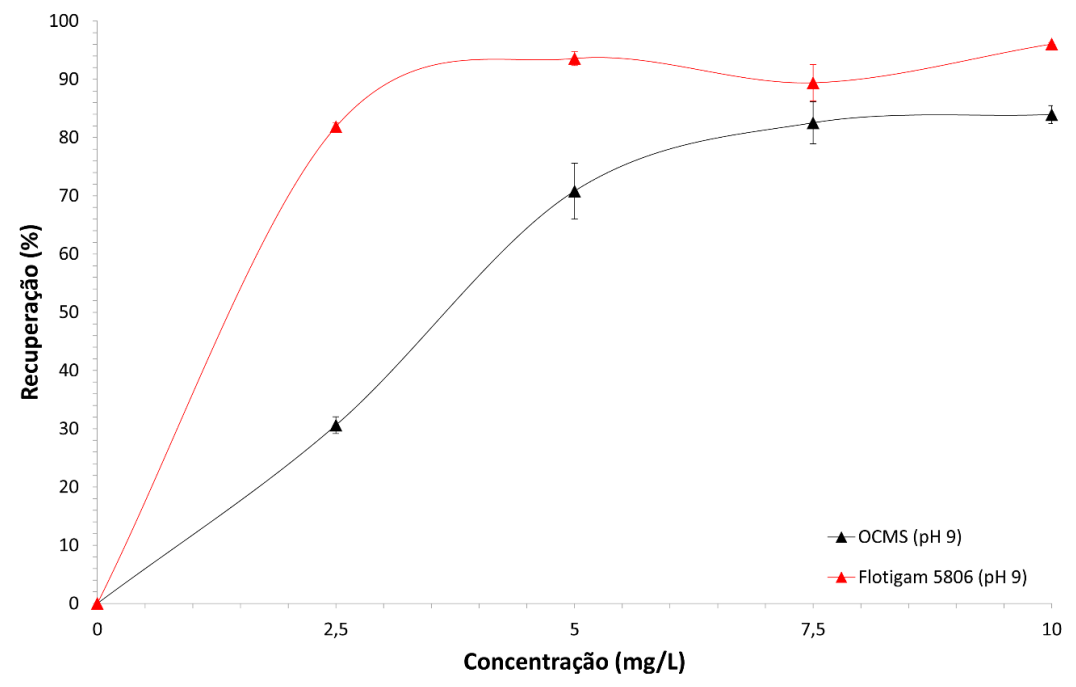

Figura 2. Recuperação de apatita em função da dosagem dos coletores OCMS e Flotigam 5806 em pH 9. 


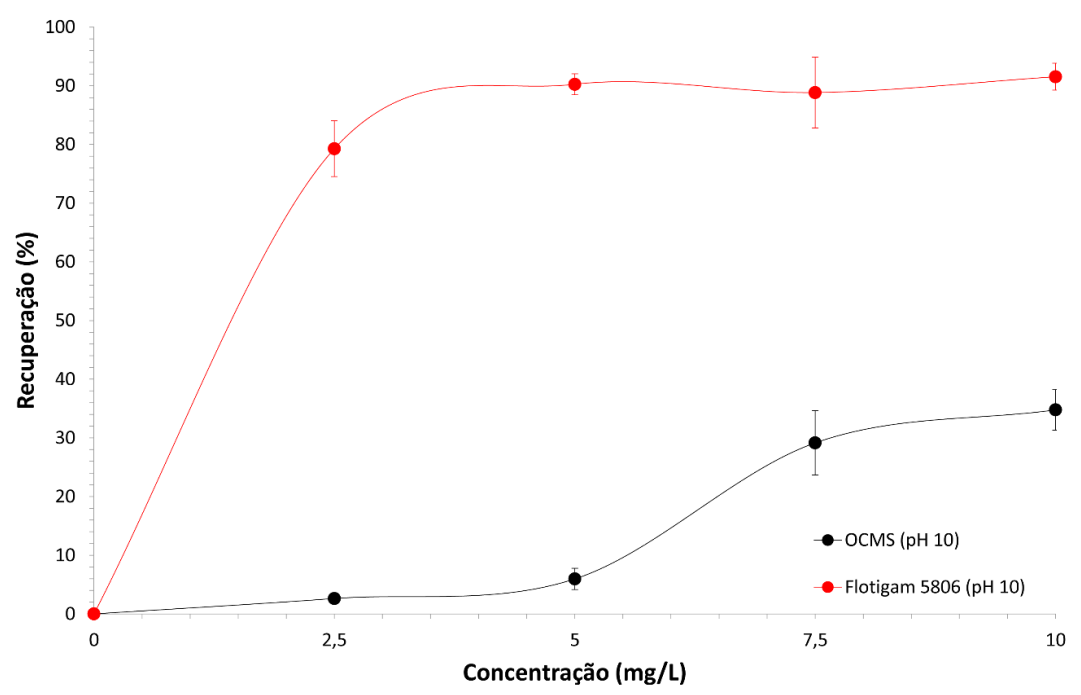

Figura 3. Recuperação de apatita em função da dosagem dos coletores OCMS e Flotigam 5806 em pH 10.

mostram que o OCMS obteve resultados de recuperação de apatita menores que o Flotigam 5806 para todas as concentrações e pH's testados. O OCMS apresentou maiores recuperações nos $\mathrm{pH}$ 's 8 e 9, sobretudo nas concentrações 7,5 e $10,0 \mathrm{mg} / \mathrm{L}$. No pH 10 o OCMS apresentou baixa recuperação em todas as concentrações testadas.

A baixa recuperação observada para o OCM pode decorrer da sua composição em ácidos graxos, visto que a literatura ressalta o desempenho superior dos ácidos graxos insaturados sobre os saturados, no que tange a seletividade. Brandão et al. [15], que também realizaram ensaios de microflotação em tubo de Hallimond com amostras de apatita, demonstraram que os ácidos graxos insaturados apresentaram melhores resultados em detrimento aos saturados.

Uma vez que o OCM apresenta maiores concentrações de ácido láurico (saturado) e oleico (insaturado), a ação conjunta destes ácidos, assim como dos outros ácidos com teores menores na composição do óleo, pode ter influenciado para que estes resultados ficassem relativamente abaixo de outros estudos, como o de Costa [8], por exemplo, que, embora tenha verificado a ação de frutos amazônicos com valores de $\mathrm{pH}$ ligeiramente diferentes, obteve altas taxas de recuperação da apatita a partir da concentração $2,5 \mathrm{mg} / \mathrm{L}$ para a maioria dos coletores testados.

\section{CONCLUSÕES}

Verifica-se que o óleo da castanha de macaúba saponificado (OCM) apresentou boa recuperação nas concentrações 7,5 e $10,0 \mathrm{mg} / \mathrm{L}$ para os pH's 8 e 9, confirmando, dessa forma, seu potencial como coletor na microflotação de apatita. Todavia, ao ser comparado ao Flotigam 5806 (coletor que é amplamente utilizado industrialmente na flotação de rocha fosfática) o OCM apresentou recuperações menores em todos os pH's e concentrações testados.

\section{Agradecimentos}

Os autores agradecem ao apoio financeiro, imprescindível para o desenvolvimento do presente trabalho, das agências brasileiras de fomento à pesquisa CNPq, CAPES, FAPEG e FUNAPE, à empresa Anglo American Fosfatos do Brasil eà Universidade Federal de Goiás, pelo apoio dado ao mesmo.

\section{REFERÊNCIAS}

I Fonseca DS. Sumário mineral brasileiro: fosfato. Brasília: DNPM; 2014.

2 Souza A, Fonseca D. Economia mineral do Brasil: fosfato. Brasília: DNPM; 2009.

3 Monte MBM, Peres AEC. Química de superfície na flotação. In: Luz AB, Sampaio JA, França SCA. Tratamento de minérios. 5. ed. Rio de Janeiro: CETEM/MCT; 20I0. p. 399-46I.

4 Baltar CAM. Flotação no tratamento de minérios. Recife: Editora da UFPE; 2008.

5 Bulatovic SM. Handbook of flotation reagents: chemistry, theory and practice: flotation of sulfide ores. Oxford: Elsevier Science \& Technology Books; 2007. 
6 Abdel-Khalek NA, Selim KA, Abdallah MM, El-Bellihi AA, Bayoumy WA. Flotation of egyptian newly discovered fine phosphate ore of Nile Valley. In: Proceedings of the International Conference on Mining, Material and Metallurgical Engineering; 2014 August II-12; Prague, Czech Republic. Prague; 20I4. p. I5I-I58.

7 Guimarães RC, Araújo AC, Peres AEC. Reagents in igneous phosphate ores flotation. Minerals Engineering. 2005; 18(2):199-204. http://dx.doi.org/10.1016/j.mineng.2004.08.022.

8 Costa DS. Uso de óleos vegetais amazônicos na flotação de minérios fosfáticos [tese de doutorado]. Belo Horizonte: Universidade Federal de Minas Gerais; 2012.

9 Alves A, Costa D, Meireles A, Queiroz R, Peres AEC. Aplicabilidade dos óleos de maracujá (Passiflora Edulis) e babaçu (Orbignya Phalerata) na flotação de apatita, calcita e quartzo. In: Universidade Federal de Goiás. Anais do XXV Encontro Nacional de Tratamento de Minérios e Metalurgia Extrativa e VIII Meeting of the Southern Hemisphere on Mineral Technology; 2013 October 20-24; Goiânia, Brasil. Goiânia: Gráfica Pires do Rio; 20I3. p .46-52.

10 Instituto Adolfo Lutz. Métodos químicos e físicos para análise de alimentos. 4. ed. São Paulo: Instituto Adolfo Lutz; 2008.

I I Zuppa TO. Avaliação das potencialidades de plantas nativas e introduzidas no cerrado na obtenção de óleos e gorduras vegetais [dissertação de mestrado]. Goiânia: Universidade Federal de Goiás; 200 I.

12 Hiane PA, Ramos Filho MM, Ramos MIL, Macedo MLR. Bocaiuva, Acrocomia aculeata (Jacq.) Lodd., pulp and kernel oils: Characterization and fatty acid composition. Brazilian Journal of Food Technology. 2005;8(3):256-259.

13 Amaral FP, Broetto F, Batistella CB, Jorge SMA. Extração e caracterização qualitativa de óleo de polpa e amêndoas de frutos de macaúba [Acrocomia aculeata (Jacq) Lodd. Ex Mart] coletada na região de Botucatu, SP. Revista Energia na Agricultura. 20I I;26(I): I2-20. http://dx.doi.org/I0. I 7224/EnergAgric.20 I Iv26n I p I2-20.

14 Melo PG. Produção e caracterização de biodieseis obtidos a partir da oleaginosa macaúba (Acrocomia Aculeata) [dissertação de mestrado]. Uberlândia: Universidade Federal de Uberlândia; 2012.

15 Brandão PRG, Caires LG, Queiroz DSB. Vegetable lipid oil-based collectors in the flotation of apatite ores. Minerals Engineering. 1994;7(7):917-925. http://dx.doi.org/10.1016/0892-6875(94)90133-3.

Recebido em: 25 Nov. 2014

Aceito em: 9 Mar. 2015 